\title{
Secure Beamforming and Ergodic Secrecy Rate Analysis for Amplify-and-Forward Relay Networks with Wireless Powered Jammer
}

\author{
Omer Waqar, Member, IEEE, Hina Tabassum, Senior Member, IEEE and Raviraj Adve, Fellow, IEEE
}

\begin{abstract}
In this correspondence, we consider an amplify-andforward relay network in which relayed information is overheard by an eavesdropper. In order to confound the eavesdropper, a wireless-powered jammer is also considered which harvests energy from a multiple-antenna source. We proposed a new secure beamforming scheme in which beamforming vector is a linear combination of the energy beamforming (EB) and information beamforming (IB) vectors. We also present a new closed-form solution for the proposed beamforming vector which is shown to achieve a higher secrecy rate as compared to the trivial EB and IB vectors. Moreover, a tight closed-form approximation for the ergodic secrecy rate is also derived for the asymptotic regime of a large number of antennas at the source. Finally, numerical examples and simulations are provided which validate our analytical results.
\end{abstract}

Index Terms-Amplify-and-forward, ergodic secrecy rate, secure beamforming, wireless powered jammer.

\section{INTRODUCTION}

$\mathbf{O}$ WING to the benefits of the relaying architectures in increasing the network coverage and/or its performance, an overwhelming interest for wireless relay networks has been exhibited by both industry and academia over the past decade [1]. Relays play a critical role in establishing a communication between the transmitter and the receiver particularly when a direct link between these two nodes does not exist. However, due to the broadcast nature of the wireless medium, in addition to the legitimate receiver, relayed information can also be overheard by a malicious eavesdropper.

In order to safeguard the confidential message from wiretapping, physical layer security (PLS) is considered as a promising security mechanism which eliminates the drawbacks of the conventional cryptographic techniques [2]. The ultimate goal of PLS is to enhance the secrecy rate which is defined as the rate difference between the legitimate channel and the wiretap channel [3]. The significance of the secrecy rate lies in the fact that secure communications (i.e., eavesdropper is unable to decode a confidential message) is possible only when this rate is positive.

To increase the secrecy rate, one of the most effective approaches is to degrade the wiretap channel through controlled artificial noise (AN), as proposed in [4]. Following the

O. Waqar is with the Department of Engineering \& Applied Science, Thompson Rivers University (TRU), Canada. e-mail: owaqar@tru.ca.

H. Tabassum is with the Department of Electrical Engineering and Computer Science, York University, Canada. e-mail: hina@eecs.yorku.ca.

R. Adve is with the Department of Electrical \& Computer Engineering, University of Toronto, Canada. e-mail: rsadve@ece.utoronto.ca. work of [4], several strategies have been devised for wireless relay networks under the umbrella of cooperative jamming, e.g., in [5]-[7] (see [8] for a comprehensive list of works regarding cooperative jamming). In [5], Li et al. considered multiple multi-antenna relays and eavesdroppers where each relay participates in cooperative beamforming while emitting AN. In [6], jamming power allocation strategies have been investigated for an amplify-and-forward (AF) relay network in which the destination transmits an intended jamming signal via relay to confuse an eavesdropper. New closed-form expressions for the ergodic achievable secrecy rate have been derived in [7] with a multiple-antenna AF relay considering three different secure transmission schemes.

Despite the fact that in many wireless applications, the nodes are energy-constrained and have limited battery-life, most of the works including the papers mentioned above assumed that the nodes which generate jamming signals either are equipped with batteries of infinite capacities or these jammers are connected to a fixed power supply. To this end, wireless energy harvesting through radio frequency (RF) signals has emanated as a promising solution which facilitates to provide uninterruptible and controlled power to the energy-constrained jammers [9], [10] and references therein. In contrast to the existing works, we propose a new secure beamforming scheme with multiple antennas at the source and a wireless powered jammer for an AF relay network.

In particular, our main contributions are: (i) We present a new closed-form solution for the beamforming vector which maximizes the instantaneous secrecy capacity. This closedform solution provides direct insights into the impact of various system parameters on the proposed beamforming scheme. Moreover, as our proposed secure beamforming scheme is a linear combination of energy beamforming (EB) and information beamforming (IB), it achieves a higher ergodic secrecy rate (ESR) when compared to the relatively straightforward EB and IB schemes; (ii) We provide a new closed-form approximation for the ESR with a given beamforming vector and it is shown that the approximation is tight for large number of antennas at the source. Therefore, the closed-form approximation evaluates the ESR efficiently without resorting to time-consuming simulations.

Notations: $\|\mathbf{x}\|$ and $\mathbf{x}^{\dagger}$ denote the Euclidean norm and conjugate-transpose of vector $\mathbf{x}$, respectively. The norm of a complex number $z$ is denoted by $|z|$. Moreover $\mathbb{E}(\cdot)$ denotes expectation operator. $[y]^{+} \triangleq \max (y, 0)$ and $f_{X}(x)$ represents probability density function. $E_{i}(\cdot), E_{1}(\cdot)$ are exponential 
integral functions, $\ln \{\cdot\}$ is natural logarithmic function and $G_{p, q}^{m, n}[\cdot]$ is Meijer-G function [11].

\section{System Model and Signal-to-Noise Ratio}

We consider a five-node network which comprises of a source (S), a trusted variable-gain relay $(\mathbf{R})$, an external jammer (J), legitimate destination (D) and an eavesdropper (E). All nodes are equipped with a single-antenna except $\mathbf{S}$ which has $N(N \geq 1)$ antennas. Moreover, all nodes have access to fixed power supplies except $\mathbf{J}$ which harvests energy using $\mathrm{RF}$ signals originating from $\mathbf{S}$. Furthermore, it is assumed that both $\mathbf{R}$ and $\mathbf{J}$ operate in half-duplex mode with AF relaying protocol is adopted at $\mathbf{R}$. Moreover, as in [12] we consider that the AN from $\mathbf{J}$ is either nulled out at $\mathbf{D}$ through cooperation or it does not reach to $\mathbf{D}$ due to an obstacle between $\mathbf{J}$ and D. Similarly, the direct link between $\mathbf{S}$ and $\mathbf{D}$ (and $\mathbf{E}$ ) does not exist due to poor channel conditions. We consider block fading channels i.e., all channels remain constant for a block time $T$ and then change independently. The communication takes place using two phases each of length $T / 2$.

During phase 1, the signals received at $\mathbf{J}\left(y_{J}\right)$ and $\mathbf{R}\left(y_{R}\right)$ are, respectively, given as follows

$$
\begin{aligned}
& y_{J}=\sqrt{\frac{P_{s} L_{c}}{d_{S J}^{\alpha}}} \mathbf{h}_{S J}^{\dagger} \mathbf{w} x_{S}+n_{J}, \\
& y_{R}=\sqrt{\frac{P_{s} L_{c}}{d_{S R}^{\alpha}}} \mathbf{h}_{S R}^{\dagger} \mathbf{w} x_{S}+n_{R},
\end{aligned}
$$

where $x_{S}$ is an information signal transmitted from $\mathbf{S}$ with $\mathbb{E}\left\{\left|x_{S}\right|^{2}\right\}=1$ and $P_{s}$ is the transmit power of $\mathbf{S}$. Moreover, w is $N \times 1$ unit-norm beamforming vector. $d_{i j}$ denotes the distance between the $i$-th and $j$-th node where $i, j \in(\mathbf{S}, \mathbf{R}, \mathbf{J}$, $\mathbf{D}$ and $\mathbf{E}) . L_{c}$ and $\alpha$ represent path-loss constant and a pathloss exponent, respectively. Furthermore, $\mathbf{h}_{S J}$ and $\mathbf{h}_{S R}$ are $N \times 1$ channel vectors for the $\mathbf{S}$ to $\mathbf{J}$ and $\mathbf{S}$ to $\mathbf{R}$ links, respectively with mutually independent complex entries following complex circularly symmetric Gaussian (CSG) distributions of zero mean and unit variance. Moreover, $n_{i}$ denotes the additive white Gaussian noise (AWGN) at $i$-th node following complex CSG distribution with zero mean and variance $\sigma_{n}^{2}$.

During phase 2, the signal transmitted by $\mathbf{J}$ is $x_{J}=\sqrt{P_{J}} v$, where $P_{J}$ is the transmit power of $\mathbf{J}$ and $v$ is a unit-power AN signal. Using (1) and assuming that the noise power is negligible for energy harvesting purposes, we can write

$$
x_{J}=\sqrt{\eta P_{s} L_{c} d_{S J}^{-\alpha}}\left|\mathbf{h}_{S J}^{\dagger} \mathbf{w}\right| v,
$$

where $\eta \in[0,1]$ represents an energy conversion efficiency. Moreover, the signal transmitted by $\mathbf{R}$ is $x_{R}=\beta y_{R}$, where $\beta$ is an amplification gain factor which satisfies $\mathbb{E}\left\{\left|x_{R}\right|^{2}\right\}=$ $P_{R}$. Therefore, we can write

$$
\beta^{2}=\frac{P_{R}}{P_{S} L_{c} d_{S R}^{-\alpha}\left|\mathbf{h}_{S R}^{\dagger} \mathbf{w}\right|^{2}+\sigma_{n}^{2}},
$$

where $P_{R}$ denotes the transmit power of $\mathbf{R}$. The signals received at $\mathbf{D}\left(y_{D}\right)$ and $\mathbf{E}\left(y_{E}\right)$ are, respectively, given as

$$
y_{D}=\beta \sqrt{\frac{P_{s} L_{c}^{2}}{\left(d_{S R} d_{R D}\right)^{\alpha}}}\left(\mathbf{h}_{S R}^{\dagger} \mathbf{w}\right) h_{R D} x_{S}+\frac{\beta h_{R D} n_{R}}{\sqrt{L_{c} d_{R D}^{\alpha}}}+n_{D},
$$

$$
\begin{array}{r}
y_{E}=\beta \sqrt{\frac{P_{s} L_{c}^{2}}{\left(d_{S R} d_{R E}\right)^{\alpha}}}\left(\mathbf{h}_{S R}^{\dagger} \mathbf{w}\right) h_{R E} x_{S}+\frac{\beta h_{R E} n_{R}}{\sqrt{L_{c} d_{R E}^{\alpha}}} \\
+\sqrt{\frac{\eta P_{s} L_{c}^{2}}{\left(d_{S J} d_{J E}\right)^{\alpha}}}\left|\mathbf{h}_{S J}^{\dagger} \mathbf{w}\right| h_{J E} v+n_{E},
\end{array}
$$

where $h_{R D}, h_{J E}$ and $h_{R E}$ denote the channel coefficients for the $\mathbf{R}$ to $\mathbf{D}, \mathbf{J}$ to $\mathbf{E}$ and $\mathbf{R}$ to $\mathbf{E}$ links, respectively, each following complex CSG distribution with zero mean and unit variance. Substituting (4) into (5) and (6) and doing some manipulations, the instantaneous signal-to-noise ratios (SNRs) at $\mathbf{D}\left(\gamma_{D}\right)$ and $\mathbf{E}\left(\gamma_{E}\right)$ are, respectively, given as

$$
\begin{gathered}
\gamma_{D}=\frac{\gamma_{S R} \gamma_{R D}}{\gamma_{S R}+\gamma_{R D}+1}<\gamma_{D}^{\mathrm{ub}}=\frac{\gamma_{S R} \gamma_{R D}}{\gamma_{S R}+\gamma_{R D}}, \\
\gamma_{E}=\frac{\gamma_{S R} \gamma_{R E}}{\gamma_{R E}+\left(\gamma_{S R}+1\right)\left(\gamma_{J E}+1\right)},
\end{gathered}
$$

where the superscript 'ub' stands for upper-bound, $\gamma_{S R} \triangleq$ $K_{1}\left|\mathbf{h}_{S R}^{\dagger} \mathbf{w}\right|^{2}, \gamma_{R D} \triangleq K_{2} X_{R D}, \gamma_{J E} \triangleq K_{3}\left|\mathbf{h}_{S J}^{\dagger} \mathbf{w}\right|^{2} X_{J E}$, $\gamma_{R E} \triangleq K_{4} X_{R E}, X_{R D} \triangleq\left|h_{R D}\right|^{2}, X_{J E} \triangleq\left|h_{J E}\right|^{2}$ and $X_{R E} \triangleq\left|h_{R E}\right|^{2}$. Furthermore, $K_{1} \triangleq\left(P_{S} L_{c} d_{S R}^{-\alpha}\right) / \sigma_{n}^{2}, K_{2} \triangleq$ $\left(P_{R} L_{c} d_{R D}^{-\alpha}\right) / \sigma_{n}^{2}, K_{3} \triangleq\left(\eta P_{S} L_{c}^{2} d_{S J} d_{J E}\right)^{-\alpha} / \sigma_{n}^{2}$ and $K_{4} \triangleq$ $\left(P_{R} L_{c} d_{R E}^{-\alpha}\right) / \sigma_{n}^{2}$. The exact instantaneous secrecy capacity $(C)$ is given as

$$
C=\frac{1}{2}\left[C_{D}-C_{E}\right]^{+},
$$

where $C_{D} \triangleq \log _{2}\left(1+\gamma_{D}\right), C_{E} \triangleq \log _{2}\left(1+\gamma_{E}\right)$ and the factor of $1 / 2$ is due to the fact that both $\mathbf{R}$ and $\mathbf{J}$ operate in half-duplex mode.

\section{SECRECy ANALYSis}

\section{A. Secure Beamforming}

In this subsection, we present a new secure beamforming scheme and derive a closed-form solution for the near optimal beamforming vector assuming a large number of antennas at $\mathbf{S}$. To this end, we formulate the following optimization problem

$$
P_{0}: \quad \arg \max _{\mathbf{w}} C \quad \text { s.t. }\|\mathbf{w}\|^{2}=1 .
$$

The problem $P_{0}$ is non-convex, hence a time-consuming $N$ dimensional search is required. Motivated by this, in the following we provide a new closed-form solution of (10) which renders not only an efficient evaluation for the near optimal beamforming vector (i.e., that maximizes an approximation of the objective function) but also provides a way to characterize a closed-form approximation of the ergodic secrecy rate (as depicted in subsection 3B).

Proposition 1. In the asymptotic large antenna regime, i.e., as $N \rightarrow \infty$, the near optimal solution $\left(\mathbf{w}^{o}\right)$ for problem given in (10) is a linear combination of EB and IB vectors and is given as

$$
\mathbf{w}^{o}=\bar{t} \mathbf{w}_{I}+\sqrt{1-\bar{t}^{2}} \mathbf{w}_{E},
$$

where $\mathbf{w}_{I}$ and $\mathbf{w}_{E}$ represent the IB and EB vectors, respectively, i.e., $\quad \mathbf{w}_{I} \triangleq \mathbf{h}_{S R} /\left\|\mathbf{h}_{S R}\right\|, \quad \mathbf{w}_{E} \triangleq$ $\mathbf{h}_{S J} /\left\|\mathbf{h}_{S J}\right\|, \quad \bar{t} \triangleq \sqrt{\left(-1+\sqrt{1+B_{0}}\right) / B_{0}}$ and $B_{0} \triangleq$ $N\left(K_{1}\left(\gamma_{R E} / \gamma_{R D}\right)-K_{3} X_{J E}\right) /\left(K_{3} N X_{J E}+\gamma_{R E}\right)$. 
Proof: See Appendix A.

It is worth pointing out here that Proposition 1 provides some interesting insights, e.g., when $B_{0} \rightarrow-1, \mathbf{w}^{o} \rightarrow \mathbf{w}_{I}$ and $\mathbf{w}^{o} \rightarrow \mathbf{w}_{E}$ for $B_{0} \rightarrow \infty$. Similarly when $B_{0} \rightarrow 0, \bar{t} \rightarrow 1 / \sqrt{2}$ which implies that $\mathbf{S}$ achieves a perfect balance between the IB and EB.

\section{B. Ergodic Secrecy Rate}

From [7] and using (9), $\mathbb{E}(C)$ provides the ergodic secrecy capacity, however an exact closed-form expression for $\mathbb{E}(C)$ appears to be intractable. Nevertheless, we use the following tight lower bound which is known as $\operatorname{ESR}(\bar{C})$ [7]

$$
\mathbb{E}(C) \geq \bar{C}=\frac{1}{2}\left[\mathbb{E}\left(C_{D}\right)-\mathbb{E}\left(C_{E}\right)\right]^{+} .
$$

As it clear that the problem of finding a closed-form for (12) has been reduced obtaining the closed-form expressions for the $\mathbb{E}\left(C_{D}\right)$ and $\mathbb{E}\left(C_{E}\right)$. However, it is still challenging to find the closed-form expressions due to the intricate expressions given in (7) and (8). We present these closed-form expressions in the following proposition.

Proposition 2. In the asymptotic large antenna regime, i.e., as $N \rightarrow \infty$ and for given value of ' $t$ ' the tight closed-form approximations for $\mathbb{E}\left(C_{D}\right)$ and $\mathbb{E}\left(C_{E}\right)$ are given in (22) and (25), respectively.

\section{Proof: See Appendix B.}

\section{NumericAl ExAmPles AND Simulations}

In this section, we provide numerical examples with $\eta=0.8$ and $L_{C}=0.1$. The remaining system parameters are given in the captions of each figure. To begin with, we investigate the impact of beamforming vector, $\mathbf{w}$, on the instantaneous secrecy capacity in Fig. 1. It is shown in Fig. 1 that when the channel condition of $\mathbf{R}$ to $\mathbf{E}$ link is better than that of the $\mathbf{R}$ to $\mathbf{D}$ link, $\mathbf{S}$ tends to beamform more energy towards $\mathbf{J}$ as compared to beamform information signal towards $\mathbf{R}$ to confound $\mathbf{E}$. It is interesting to see that the role of AN at $\mathbf{J}$ is very important even when $\mathbf{R}$ to $\mathbf{D}$ link is better than the $\mathbf{R}$ to $\mathbf{E}$ link except in the extreme case when the channel amplitude of $\mathbf{R}$ to $\mathbf{E}$ link approaches zero. In this case, $\mathbf{S}$ tends to beamform towards $\mathbf{R}$ instead of $\mathbf{J}$. Moreover, we notice that the secrecy capacity varies significantly with ' $t$ ' and its optimal value is quite close to that obtained through Proposition 1. Furthermore, we investigate the impact of transmission powers $\left(P_{S}\right.$ and $\left.P_{R}\right)$ on $\bar{t}$ in Fig. 2. We observe that $\bar{t}$ increases with $P_{R}$, approaching beams of equal strength (i.e., $\bar{t} \rightarrow 1 / \sqrt{2}$ ) and it decreases with $P_{S}$. This observation is also in agreement with Proposition 1, thus verifies (11).

In Fig. 3, the ergodic secrecy capacity is plotted using Monte Carlo simulations corresponding to (9) upon averaging over $10^{5}$ channel realizations. It is clear from Fig. 3 that our derived approximation in Proposition 2 is tight with these simulations for various values of the transmission powers and ' $t$ '. Moreover, Fig. 3 also shows that our proposed beamforming scheme achieves a higher ESR as compared to the 'no-jammer' case as well as the trivial EB and IB schemes. It is worth pointing out here that global knowledge of perfect

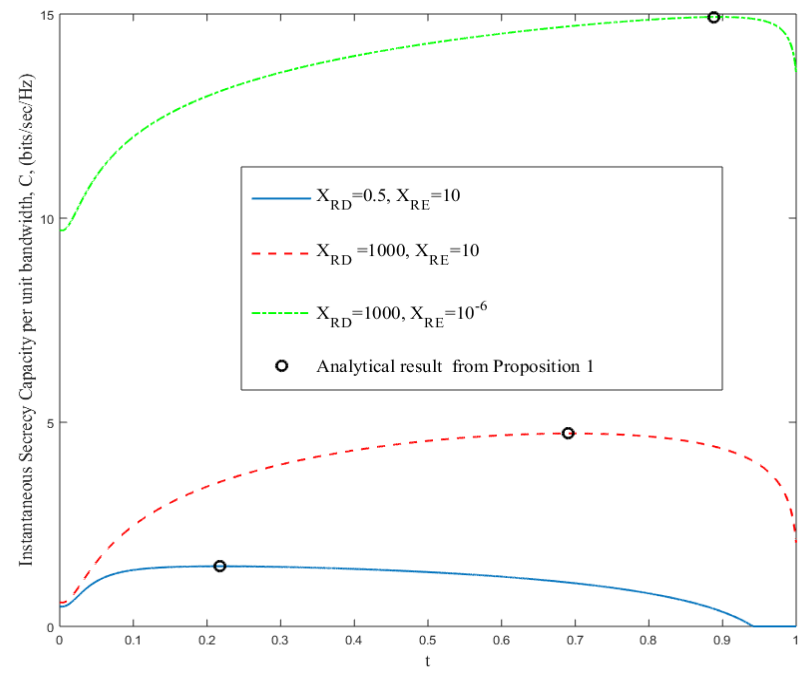

Fig. 1. Plot of instantaneous secrecy capacity using (9) for various values of t. $d_{S R}=d_{S J}=d_{R D}=d_{R E}=d_{J E}=100 \mathrm{~m}, \alpha=3, P_{S}=P_{R}=30$ $\mathrm{dBm}, \sigma_{n}^{2}=-110 \mathrm{dBm}$ and $N=200 . X_{J E}=1, \mathbf{h}_{S R}$ and $\mathbf{h}_{S J}$ are generated in MATLAB in same sequence with rng (10).

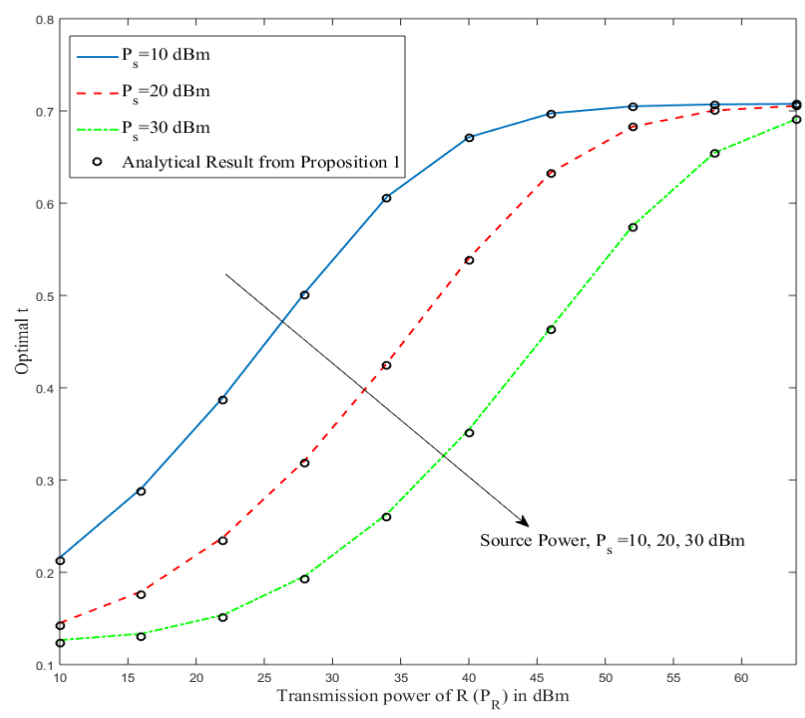

Fig. 2. Plot of instantaneous secrecy capacity using (9) for various values of $P_{R}$ and $P_{S} . d_{S R}=d_{S J}=d_{R D}=20 \mathrm{~m}, d_{R E}=30 \mathrm{~m}, d_{J E}=10 \mathrm{~m}$, $\alpha=3, \sigma_{n}^{2}=-80 \mathrm{dBm}$ and $N=500 . X_{R E}=X_{R D}=1, X_{J E}=0.82$, $\mathbf{h}_{S R}$ and $\mathbf{h}_{S J}$ are generated in MATLAB in same sequence with rng (100).

instantaneous channel state information (CSI) including that of the wiretap links (as considered in [13]) is required to calculate ' $t$ ' in both Fig. 1 and Fig. 2. However in Fig. 3, we took $t=0.35$ by replacing random variables (RVs) in $B_{0}$ by their respective averages, thus the value of ' $t$ ' remains fixed for all channel realizations (therefore only statistical knowledge of CSI for $\mathbf{R}$ to $\mathbf{E}$ and $\mathbf{R}$ to $\mathbf{D}$ links is required). Interestingly, even for a fixed ' $t$ ', the ESR is almost equal to the case in which ' $t$ ' is selected optimally for each channel realization. 


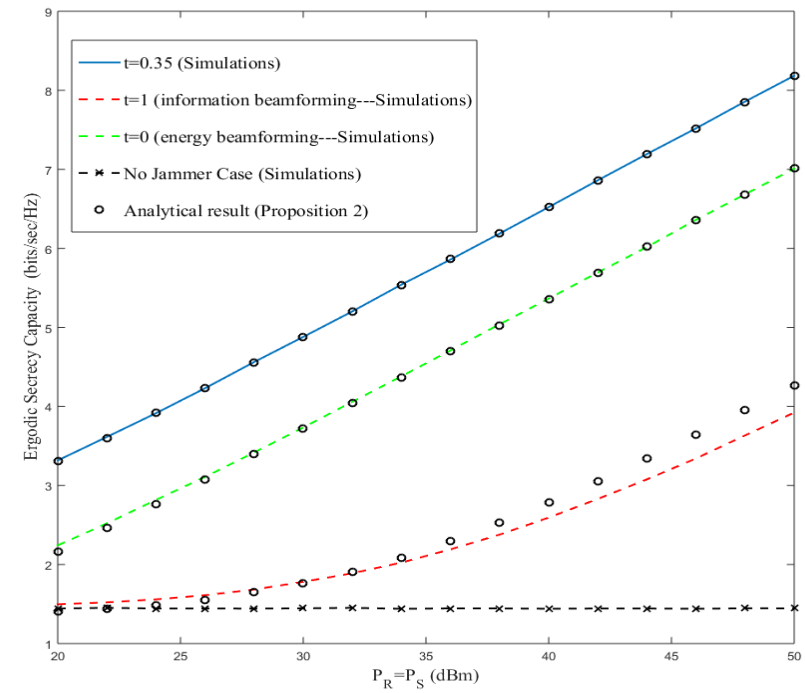

Fig. 3. Plot of ergodic secrecy capacity through simulations and ESR using (12) for various values of $P_{R}$ and $P_{S} . d_{S R}=d_{S J}=50 \mathrm{~m}, d_{R D}=$ $30 \mathrm{~m}, d_{R E}=60 \mathrm{~m}, d_{J E}=40 \mathrm{~m}, \alpha=2.7, \sigma_{n}^{2}=-80 \mathrm{dBm}$ and $N=300$.

\section{CONCLUSion}

In this correspondence, we proposed a secure beamforming scheme which is shown to achieve a higher ergodic secrecy rate compared to the trivial information and energy beamforming schemes. Moreover, we present a new closedform solution for the proposed beamforming vector which maximizes the ergodic secrecy capacity. We also derived a new closed-form approximation for the ergodic secrecy rate with a given beamforming vector. We observed that, in general, the source tends to transmit a stronger beam towards the wireless powered jammer as compared to the relay to maximize the ergodic secrecy rate except for the scenario in which the relay to eavesdropper channel is extremely weak.

\section{APPENDIX A}

\section{PROOF OF PROPOSITION 1}

Although the objective function of (10) is considerably different from that given in [14], w admits the same generic form $^{1}$ and for $N \rightarrow \infty$, we can have

$$
\mathbf{w}=t \mathbf{w}_{I}+\sqrt{1-t^{2}} \mathbf{w}_{E},
$$

where $t \in[0,1]$. Substituting (13) into the upper bound of (7) we can write

$$
\gamma_{D} \simeq \widetilde{\gamma}_{D}(t)=\frac{\widetilde{\gamma}_{S R}(t) \gamma_{R D}}{\widetilde{\gamma}_{S R}(t)+\gamma_{R D}},
$$

where leveraging law of large numbers we put $\left\|\mathbf{h}_{S R}\right\|^{2} \backsim$ $N$ and can write $\widetilde{\gamma}_{S R}(t) \triangleq K_{1}\left[N t^{2}+\left(1-t^{2}\right) Z_{S R}\right]$ with $Z_{S R} \triangleq\left|\mathbf{h}_{S R}^{\dagger} \mathbf{w}_{\mathbf{E}}\right|^{2}$ is an exponential RV of unit mean [14]. Using a similar approach as adopted in [15], (8) can be upperbounded as

\footnotetext{
${ }^{1}$ It is worth mentioning here that only the generic form of $\mathbf{w}$ remains same, our final solution is different from that of [14] due to significant difference in the objective functions.
}

$$
\gamma_{E}<\min \left(\gamma_{S R}, \frac{\gamma_{R E}}{\gamma_{J E}}\right)
$$

Now substitute (13) into (15) and using again $\left\|\mathbf{h}_{S J}\right\|^{2} \simeq N$, we can write

$$
\gamma_{E} \backsim \widetilde{\gamma}_{E}(t)=\min \left(\widetilde{\gamma}_{S R}(t), \frac{\gamma_{R E}}{\widetilde{\gamma}_{J E}(t)}\right),
$$

where $\widetilde{\gamma}_{J E}(t) \triangleq K_{3}\left[N\left(1-t^{2}\right)+t^{2} Z_{S J}\right] X_{J E}$ and $Z_{S J} \triangleq$ $\left|\mathbf{h}_{S J}^{\dagger} \mathbf{w}_{\mathbf{I}}\right|^{2}$ is an exponential RV of unit mean. Now with $\widetilde{\gamma}_{D}(t) \gg 1$, the approximation of (9) can be written as

$$
\widetilde{C}(t)=\frac{1}{2}\left[\log _{2}\left(\widetilde{\gamma}_{D}(t)\right)-\log _{2}\left(1+\widetilde{\gamma}_{E}(t)\right)\right]^{+} .
$$

Furthermore, using (17) and after some manipulation, we can reformulate the problem $P_{0}$ as

$P_{1}: \bar{t}=\arg \min _{t}\left[\min \left(g_{1}(t), g_{2}(t)\right)\right]$, s.t. $0 \leq t \leq 1$,

where $g_{1}(t) \triangleq \frac{1+\widetilde{\gamma}_{S R}(t)}{\widetilde{\gamma}_{D}(t)}$ and $g_{2}(t) \triangleq$ $\frac{1}{\widetilde{\gamma}_{D}(t)}\left[1+\frac{\gamma_{R E}}{\widetilde{\gamma}_{J E}(t)}\right]$. It is interesting to note that for $g_{1}(t) \leq g_{2}(t)$, we have $\widetilde{\gamma}_{D}(t) \leq \widetilde{\gamma}_{E}(t)$ and $\widetilde{C}(t)$ is always equal to zero $\forall t$. Therefore, problem $P_{1}$ can be simplified as

$$
P_{2}: \bar{t}=\arg \min _{t} g_{2}(t), \text { s.t. } \quad 0 \leq t \leq 1
$$

Now take first derivative of the objective function given in (19) with respect to ' $t$ ', (note that $Z_{S R}$ and $Z_{S J}$ related terms in $\widetilde{\gamma}_{S R}(t)$ and $\widetilde{\gamma}_{J E}(t)$ can be ignored as $N \rightarrow \infty$ ), substitute $t^{2}=y$ and after some manipulation, we get

$$
B_{0} y^{2}+2 y-1=0
$$

$B_{0} \triangleq N\left(K_{1}\left(\gamma_{R E} / \gamma_{R D}\right)-K_{3} X_{J E}\right) /\left(K_{3} N X_{J E}+\gamma_{R E}\right)$ with $B_{0} \in[-1, \infty)$. Using the quadratic formula, we get the following two roots for (20)

$$
y_{1}=\frac{-1-\sqrt{1+B_{0}}}{B_{0}} \text { and } y_{2}=\frac{-1+\sqrt{1+B_{0}}}{B_{0}} .
$$

Note that $y_{1} \geq 1$ for $-1 \leq B_{0}<0$ and $y_{1}<0$ for $B_{0}>0$. Moreover for $B_{0} \geq-1$, we have $0 \leq y_{2} \leq 1$, therefore we get unique minima for $P 2$ at $\bar{t}=\sqrt{y_{2}}$.

\section{APPENDIX B}

\section{ProOF OF PROPOSITION 2}

\section{Derivation of $\mathbb{E}\left(C_{D}\right)$ :}

By adopting approach as in [16], using (7) and $\widetilde{\gamma}_{S R}(t)$ from Appendix A, we can write (22). Given the fact that for any $\mathrm{RV} X, \mathbb{E}(\ln \{1+X\})=\int_{0}^{\infty} \ln \{1+x\} f_{X}(x) d x$, therefore using eq. (2.5.2.1) of [11], the identity $-E_{i}(-x)=E_{1}(x)$ and after some manipulation, we get (23) and (24)

In case for $\lambda_{D}=\mu_{D}$, using eq. (2.5.2.11) of [11] we can replace (24) with

$$
C_{D_{3}}=G_{3,2}^{1,3}\left[\mu_{D} \mid \begin{array}{c}
-1,1,1 \\
1,0
\end{array}\right] .
$$

Now placing (23) and (24) or (28) into (22), we get the desired result. 


$$
\mathbb{E}\left(C_{D}\right) \simeq \frac{1}{\ln (2)}[\underbrace{\mathbb{E}\left(\ln \left\{1+\mu_{D} Z_{S R}\right\}\right)}_{C_{D_{1}}}+\underbrace{\mathbb{E}\left(\ln \left\{1+K_{2} X_{R D}\right\}\right)}_{C_{D_{2}}}-\underbrace{\mathbb{E}\left(\ln \left\{\left(1+\mu_{D} Z_{S R}+\lambda_{D} X_{R D}\right)\right\}\right)}_{C_{D_{3}}}],
$$

where $\mu_{D} \triangleq \frac{K_{1}\left(1-t^{2}\right)}{1+K_{1} N t^{2}}$ and $\lambda_{D} \triangleq \frac{K_{2}}{1+K_{1} N t^{2}}$. Moreover,

$$
\begin{gathered}
C_{D_{1}}=\exp \left(\frac{1}{\mu_{D}}\right) E_{1}\left(\frac{1}{\mu_{D}}\right) \quad \text { and } C_{D_{2}}=\exp \left(\frac{1}{K_{2}}\right) E_{1}\left(\frac{1}{K_{2}}\right) . \\
C_{D_{3}}=\frac{1}{\lambda_{D}-\mu_{D}}\left[\lambda_{D} \exp \left(\frac{1}{\lambda_{D}}\right) E_{1}\left(\frac{1}{\lambda_{D}}\right)-\mu_{D} \exp \left(\frac{1}{\mu_{D}}\right) E_{1}\left(\frac{1}{\mu_{D}}\right)\right] \quad \text { for } \lambda_{D} \neq \mu_{D} .
\end{gathered}
$$

$$
\mathbb{E}\left(C_{E}\right) \simeq \frac{1}{\ln (2)}[\underbrace{\mathbb{E}\left(\ln \left\{1+K_{4} X_{R E}+\mu_{E} X_{J E}\right\}\right)}_{C_{E_{1}}}-\underbrace{\mathbb{E}\left(\ln \left\{\left(1+\mu_{E} X_{J E}+\lambda_{E} X_{R E}\right)\right\}\right)}_{C_{E_{2}}}],
$$

where $\mu_{E} \triangleq K_{3}\left[N\left(1-t^{2}\right)+t^{2}\right]$ and $\lambda_{E} \triangleq \frac{K_{4}}{1+K_{1}\left[N t^{2}+\left(1-t^{2}\right)\right]}$. Moreover,

$$
\begin{array}{ll}
C_{E_{1}}=\frac{1}{\mu_{E}-K_{4}}\left[\mu_{E} \exp \left(\frac{1}{\mu_{E}}\right) E_{1}\left(\frac{1}{\mu_{E}}\right)-K_{4} \exp \left(\frac{1}{K_{4}}\right) E_{1}\left(\frac{1}{K_{4}}\right)\right] & \text { for } K_{4} \neq \mu_{E} . \\
C_{E_{2}}=\frac{1}{\lambda_{E}-\mu_{E}}\left[\lambda_{E} \exp \left(\frac{1}{\lambda_{E}}\right) E_{1}\left(\frac{1}{\lambda_{E}}\right)-\mu_{E} \exp \left(\frac{1}{\mu_{E}}\right) E_{1}\left(\frac{1}{\mu_{E}}\right)\right] & \text { for } \lambda_{E} \neq \mu_{E} .
\end{array}
$$

\section{Derivation of $\mathbb{E}\left(C_{E}\right)$ :}

Again adopting the approach of [16] and after few manipulation, we can write (25). The closed-form expression for (25) appears to be intractable, thus we replaced RVs $Z_{S R}=Z_{S J}$ with their respective means (which are unity). As the remaining derivation for $\mathbb{E}\left(C_{E}\right)$ is similar to that of $\mathbb{E}\left(C_{D}\right)$, for sake of brevity we skipped the steps. Furthermore, for $K_{4}=\mu_{E}$ or $\lambda_{E}=\mu_{E}$, we can replace (26) or (27) accordingly with

$$
C_{E_{1}}\left(\text { or } C_{E_{2}}\right)=G_{3,2}^{1,3}\left[\mu_{E} \mid \begin{array}{c}
-1,1,1 \\
1,0
\end{array}\right] .
$$

Now the evaluation for $\mathbb{E}\left(C_{E}\right)$ can be done using (25).

\section{REFERENCES}

[1] Y. Hua, D. W. Bliss, S. Gazor, Y. Rong, and Y. Sung, "Guest editorial: Theories and methods for advanced wireless relays - Issue II," IEEE J. Sel. Areas Commun., vol. 31, no. 8, pp. 1361-1367, 2013.

[2] A. Mukherjee, S. A. A. Fakoorian, J. Huang, and A. L. Swindlehurst, "Principles of physical layer security in multiuser wireless networks: A survey," IEEE Commun. Surv. Tut., vol. 16, no. 3, pp. 1550-1573, 2014

[3] X. Chen, J. Chen, and T. Liu, "Secure transmission in wireless powered massive MIMO relaying systems: Performance analysis and optimization," IEEE Trans. Veh. Technol., vol. 65, no. 10, pp. 8025-8035, 2016.

[4] S. Goel and R. Negi, "Guaranteeing secrecy using artificial noise," IEEE Trans. Wireless Commun., vol. 7, no. 6, pp. 2180-2189, 2008.

[5] Q. Li, Y. Yang, W. Ma, M. Lin, J. Ge, and J. Lin, "Robust cooperative beamforming and artificial noise design for physical-layer secrecy in AF multi-antenna multi-relay networks," IEEE Trans. Signal Process., vol. 63, no. 1, pp. 206-220, 2015.

[6] K. Park, T. Wang, and M. Alouini, "On the jamming power allocation for secure amplify-and-forward relaying via cooperative jamming," IEEE $J$. Sel. Areas Commun., vol. 31, no. 9, pp. 1741-1750, 2013.

[7] R. Zhao, Y. Huang, W. Wang, and V. K. N. Lau, "Ergodic achievable secrecy rate of multiple-antenna relay systems with cooperative jamming," IEEE Trans. Wireless Commun., vol. 15, no. 4, pp. 2537-2551, 2016.
[8] F. Jameel, S. Wyne, G. Kaddoum, and T. Q. Duong, "A comprehensive survey on cooperative relaying and jamming strategies for physical layer security," IEEE Commun. Surveys Tutorials, vol. 21, no. 3, pp. 27342771, 2019.

[9] H. Xing, K. Wong, Z. Chu, and A. Nallanathan, "To harvest and jam: A paradigm of self-sustaining friendly jammers for secure AF relaying," IEEE Trans. Signal Process., vol. 63, no. 24, pp. 6616-6631, 2015.

[10] Z. Mobini, M. Mohammadi, and C. Tellambura, "Wireless-powered fullduplex relay and friendly jamming for secure cooperative communications," IEEE Trans. Inf. Forensics Security, vol. 14, no. 3, pp. 621-634, 2019.

[11] A. Prudnikov, Y. Brychkov, and O. Marichev, Integrals and series. Vol. 4: Direct Laplace transforms, 1992.

[12] T. M. Hoang, T. Q. Duong, N. Vo, and C. Kundu, "Physical layer security in cooperative energy harvesting networks with a friendly jammer," IEEE Wireless Commun. Lett., vol. 6, no. 2, pp. 174-177, 2017.

[13] Y. Zou, X. Wang, and W. Shen, "Optimal relay selection for physicallayer security in cooperative wireless networks," IEEE J. Sel. Areas Commun., vol. 31, no. 10, pp. 2099-2111, 2013.

[14] O. Waqar and R. Adve, "On the throughput of wireless powered communication systems with a multiple antenna bidirectional relay," IEEE Wireless Commun. Lett., vol. 8, no. 3, pp. 941-944, 2019.

[15] I. Krikidis, J. S. Thompson, S. Mclaughlin, and N. Goertz, "Max-min relay selection for legacy amplify-and-forward systems with interference," IEEE Trans. on Wireless Commun., vol. 8, no. 6, pp. 3016-3027, 2009.

[16] O. Waqar, D. C. McLernon, and M. Ghogho, "Exact evaluation of ergodic capacity for multihop variable-gain relay networks: A unified framework for generalized fading channels," IEEE Trans. Veh. Technol., vol. 59 , no. 8 , pp. 4181-4187, 2010 . 